\title{
The Use of the Artificial Immune Network Algorithm AIN in Distinguishing English Character Pattern
}

\author{
Maha Abd Alellah Mohammad \\ Mahaabd77@uomosul.edu.iq \\ Collage of computer science and Mathematics \\ University of Mosul, Mosul, Iraq
}

Received on: 07/10/2012

Accepted on: 30/01/2013

\begin{abstract}
The focus of the present research is on the issue of patterns compatibility regarding an English letter through the use of a probable research Algorithm called the Artificial Immune Network (AIN). The research clarifies the algorithm ability in patterns compatibility between the original (ideal) pattern of the letter and the deformed patterns since the Artificial Immune Network (AIN) is good for some tasks that require examples. It applies to the issues that have large (wide) areas and large variables. In addition, it can also be quickly and easily solved as well as it provides a solution that is quite near to the ideal solution of the patterns used, The data base used contain file involves data for each original (ideal) pattern of the English letter, the pattern recognition operation (template matching) provided \%94 by using Artificial Immune Network. knowing that we obtain the practical result by using MATLAB 2008.
\end{abstract}

Keywords: Natural immune system, artificial immune system, pattern recognition, clonal selection theory, Artificial immune network.

$$
\begin{aligned}
& \text { استخدام خوارزمية الثبكة المناعية الاصطناعية AIN في تمييز أنماط الحروف الإنكليزية } \\
& \text { مها عبد الإله البدراني } \\
& \text { كلية علوم الحاسوب والرياضيات } \\
& \text { جامعة الدوصل، الموصل، العرلق الهول }
\end{aligned}
$$

تاريخ قبول البحث: 2013/01/30

تاريخ استلام البحث: 2012/10/7

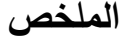

تركز الاهتمام في هذا البحث على مسألة تطابق الأنماط للحرف الإنكليزي باستخدام خوارزمية بحث

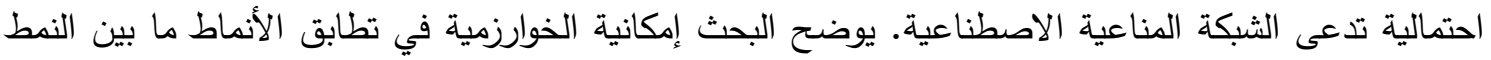

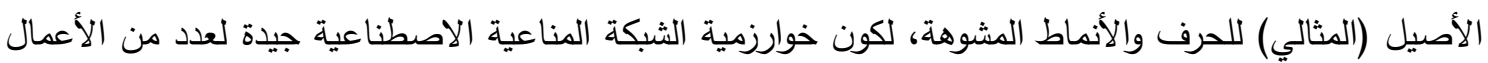

التي تتطلب الأمثلة، فهي تطبق على المسائل التي تمتلك مساحة واسعة ومتغيرات كبيرة وفي الإمكان حلها بسهولة

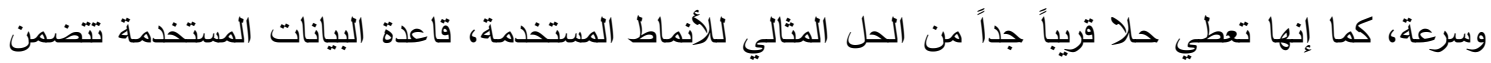

ملفاً يحتوي بيانات لكل نمط حرف انكليزي أصلي، حققت عملية تمييز أنماط الحروف الانكليزية (طريقة مطابقة
} 
القالب) باستخدام الثبكة المناعية الاصطناعية نسبة 94\% علما انه تم الحصول على النتائج عملياً باستخدام لغة .MATLAB 2008 الكلمات المفتاحية: الجهاز المناعي الطبيعي , نظام المناعة الاصطناعي, تمييز الانماط , نظرية الانتقاء النسيلي , الثبكة المناعية الاصطناعيه.

\section{1المقدمة}

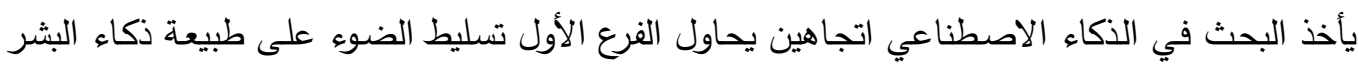

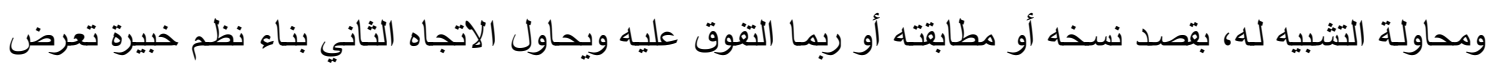
سلوك ذكي بغض النظر عن مشابهته لذكاء الإنسان[5]. وتهتم الدراسات الأخيرة ببناء أدوات ذكيه لمساعدة الإنسان في مهام معقدة مثل التشخيص الطبان إنبي، التحليل

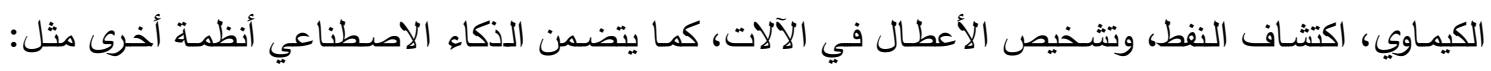

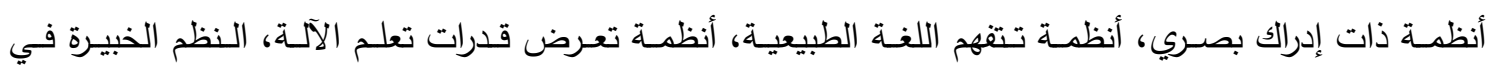
التصنيع[3]. يعمل الذكاء الاصطناعي معتمدا على مبدأ مضـاهاة التشكيلات التي يمكن بواسطته وصف الأشياء

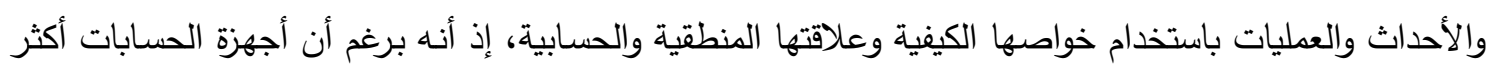

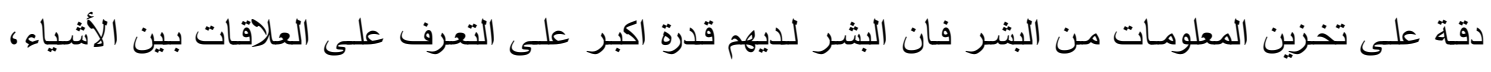

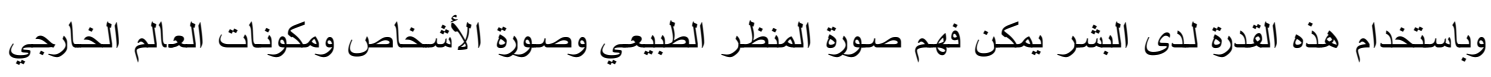
وفهم معانيها وعلاقات بعضها بالبعض ولو أمكن وضع هذه المقدرة في جهاز الحاسب لأصبح ذكيا، و برغم هذه

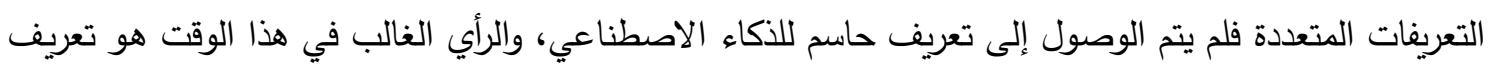

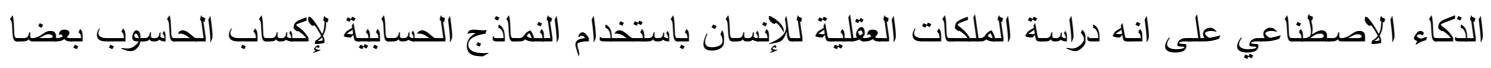
منها [13][2] 2. 2 - 2 مدف البحث

يهدف البحث إلى استخدام إحدى التقنيات الذكائية الاصطناعية وهي النظام المناعي الاصطناعي حيث

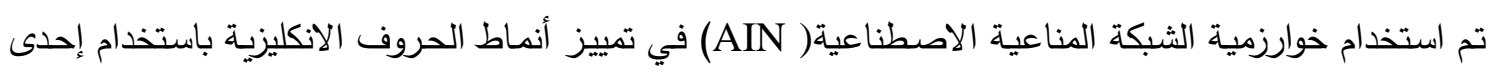

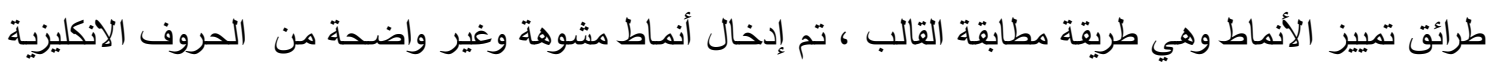

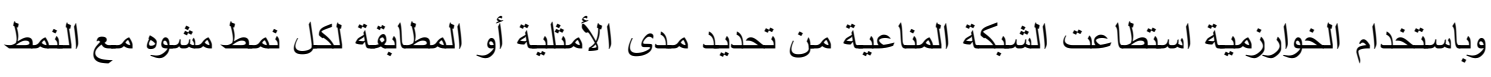

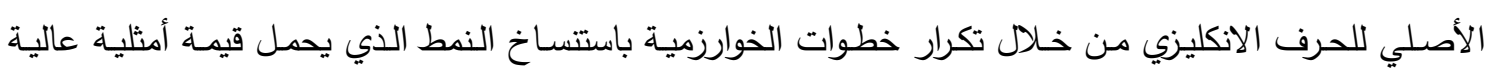
وحذف النمط الذي يمتلك قيمة أمثلية اقل من القيمة المحددة لكل نمط أصلي، برهنت النتائج قدرة خوارزمية الشبكة المناعية الاصطناعية على تمييز اغلب الأنماط المشوهة المدخلة.

3. الدراسات السابقة في تمييز أنماط الحروف يعد تمييز الأنماط من أهم المواضيع التي تثغل الباحثين في الوقت الحاضر إذ يتم الاعتماد فيها على ذئ مبرمجين أكفاء ذوي خبرة عالية لبناء نظام حاسوبي قادر على تمييز الأنماط بواسطة تقنيات وخوارزميات معينة 
كتقنيات الذكاء الاصطناعي، في بحوث سابقة تم إجراء عملية تمييز أنماط الحروف الانكليزية باستخدام الخوارزمية الجينية [1]، إذ تم اعتماد طريقة مطابقة القالب في تمييز النمط المشوه للحرف الانكليزي ومقارنته مع الحرف تهاء الانكليزي الأصلي.

C.-L. LIU, C. العالم C.-L. LIU

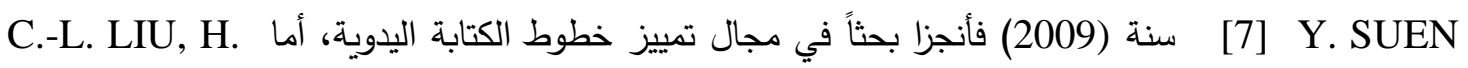
T. H. SU, T. W. 8نة SAKO

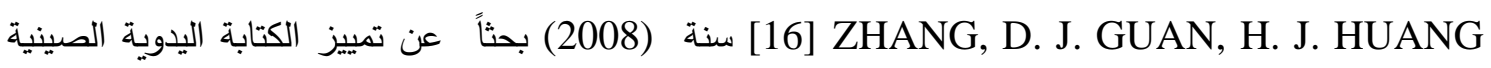
في مجال تمييز الأنماط وبطريقة التقطيع · 4. - 2 الجهاز المناعي الطبيعي

لقد تمت دراسة جهاز المناعة عند الإنسان بصورة جيدة لما يزيد عن المائة عام ولكن لا يزال هذا الجهاز لم يفهم بثكل كامل، فجهاز المناعة هو نظام دفاعي متطور يحمي المضيف من الكائنات الممرضة (الكائنات الحية

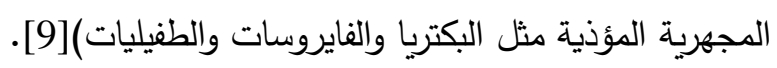

يتم تتشيط الجهاز المناعي ألتكيفي أو "المتخصص" بواسطة الجهاز المناعي الطبيعي "غير المتخصصئل الأقدم من حيث النشأة (والذي يعد آلية دفاع المضيف الأساسية ضد العوامل المسببة للمرض في معظم الكائنات

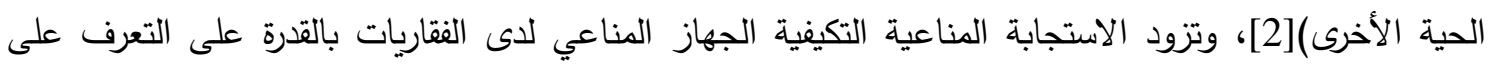

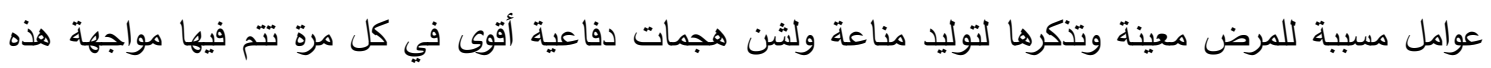
العوامل المسببة للمرض على وجه التحديد، ويشار إلى هذا النوع من المناعة باسم المناعة التكيفية نظراً لأن

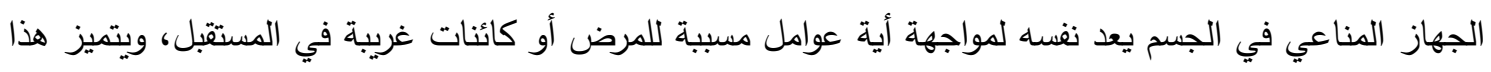
الجهاز بأنه قابل للتكيف بدرجة عالية بسبب فرط الطفرة الجسدية (حدوث مجموعة من الطفرات الجسدية المتلاحقة بشكل سريع) وعملية إعادة تثكيل اتحاد جيني غير قابلة للعكس لأجزاء من جينات مستقبلات الأجسام المضادة

\section{5. نظام المناعة الاصطناعي Artificial Immune System}

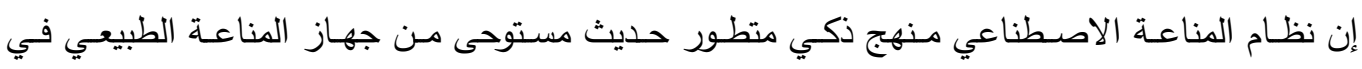
الإنسان، ظهر في التسعينات على انه فرع جديد من الحساب التطوري، إن جهاز المناعة الطبيعي معقدٌ جداً ليتم محاكاته صناعياً ولكن نجح A.B.Watkins بمحاكاة أهم وظـائف جهاز المناعـة الطبيعي فيما يخص تمييز الأنماط[13]

إن نظام المناعة الاصطناعي هو مجموعـة من خوارزميات التصنيف الذكية التي تستخدم آلية دفاع المناعة الطبيعية لأغراض تقنية قادرة على التكيف والتعلم، لذلك انتشر هذا المفهوم في تطبيقات تقنية عديدة خلال العقود الأخيرة [2].

يمكن تقسيم الخوارزميات في أنظمة AIS بثكل واسع على ثلاثة أصناف مستتدة إلى النظريات الإحيائية الملهمة منها [12]:1. الخوارزميات المبنية على المجتمع (12) (population-based). 
2. الخوارزميات المبنية على الثبكة (network-based). 3. الخوارزميات المبنية على نظرية الخطر (danger theory).

لقد تم تطبيق الأنظمة المناعية الاصطناعية بوصفها حسلا للمشكلات في مجالات واسعة، منها تمييز الأنماط، كثف الخطأ والثذوذ، تحليل البيانات، الأنظمة المعتمدة على الوكلاء، وسائل البحث والأمثلية، حماية

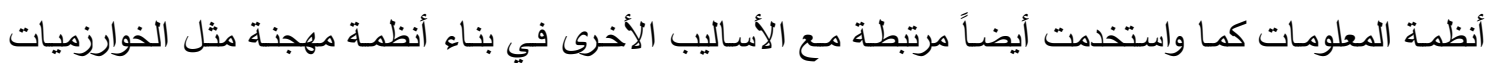

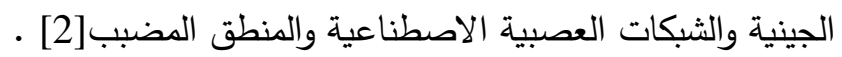

6. الخوارزميات المبنية على الثبكة Network Based Algorithm

لقد صنفت نماذج الثبكة المناعية إلى صنفين وهما:- النماذج المستمرة والنماذج المتقطعة، تم افتراض الاستجابة المناعية في النماذج المستمرة بان تكون مستمرة مقارنة مـع ما موجود في النماذج المتقطعة بان تكون ضـن خطوات زمنيـة متقطعـة. النمـاذج المستمرة موصـوفة من خـلال مجموعـة المعادلات التفاضلية، وغرضهـا الرئيسي هو في نمذجة الظاهرة الإحيائية [11][11]، وفيما يلي أصناف الخوارزميات المبنية على الثبكة:

1. نماذج الشبكة المناعية المستمرة Continuous Immune Network Models.

2. نماذج الثبكة المناعية المتقطعة Discrete Immune Network Models. 3. الشبكة المناعية الاصطناعية AIN) Artificial Immune Network (AIN)

7. تمييز الأنماط

نتيجة للضغط الذي ولدته التقانات الحديثة دعت الحاجة إلى ضرورة بناء آلات تستطيع القيام بالمهام التي يقوم بها الإنسان على نحو أدق وأسرع وبكلفة اقل مما يقوم به البشر من اجل مواكبة التطور الهائل والسريع في أنشطة الحياة المختلفة [8]. لقد حاول الإنسان تدريجيا تعليم الآلة تمييز الأثياء المختلفة ليظهر فيما بعد التمييز الآلي أو ما يعرف بتمييز الأنماط (Patterns recognition)، وهو المجال الذي يعنى بدراسة كيفية قيام الآلة بمراقبة البيئة وتعليمها تمييز الأنماط ذات الاهتمام مما يحيط بها ثم اتخاذ القرار لتصنيف هذه الأنماط إلى أصناف، والأنماط جمع نمط لئه

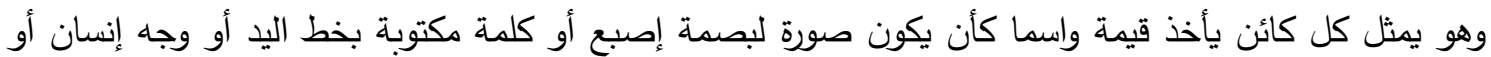
صوت إنسان، إذ لا يزال الباحثون يقدمون العديد من الدراسات والأفكار الجديدة لإيجاد التطبيقات في مجال تمثيل المعالم وتمييزها آليا[15].

اتسعت مساحة مجال تطبيق تمييز الأنماط لتثمل تطبيقات عديدة في مجالات الحياة المختلفة مثل التطبيقات الهندسية الطبية ومجالات أخرى مثل حقل الذكاء الاصطناعي (Artificial intelligence) والاستشعار عن بعد (Remote sensing) فضلا عن التسويق (Marketing) إن تصميم أي نظام لتمييز الأنماط يتطلب الانتباه إلى الأمور الآتية [5]: تعريف الأصناف، تمثيل النمط، استخلاص الخصائص، تصميم المصنف، تقييم الأداء. هالك عدة طرق لتمييز الأنماط تم اعتماد طريقة الارتباط ومطابقة القالب ( Template-Matching (Correlation Method and Templates الداخل على شكل مصفوفة ويقارن مع القوالب الموجودة في الجهاز Bit By Bit وتعطى قيمة للمقارنة[5]. 
Clonal Selection Theory نظرية الاتتقاء النسيلي

وهي خوارزمية المناعة المكتسبة التي توضح كيف تقوم خلايا T و الليمفاوية بتحسين استجابتها للمستضدات في أكثر من مرة وتدعى Affinity maturation (نضوج التقارب) هذه الخوارزمية تركز على تونى توني الصفات الداروينية[2]، حيث يتم الاختيار بالاعتماد على نسب التقارب من تفاعلات (ضد_مستضد). الاستنساخ مستوحى من انقسام الخلايا والاختلاف مستوحى من الطفرة (Somatic hyper mutation)، إن خوارزمية لإنية الانتقاء النسيلي هي الأكثر شيوعا وتطبق في مجالات التحسين وتمييز الأنماط، وفي عام 2000 تم التركيز على التى

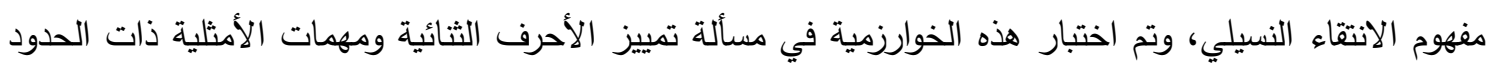

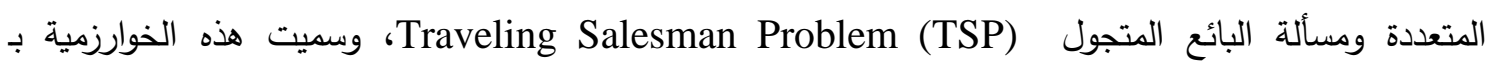

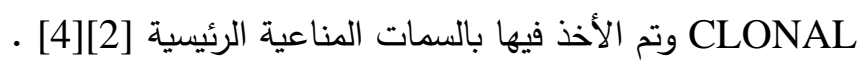

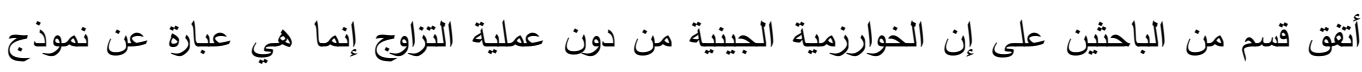

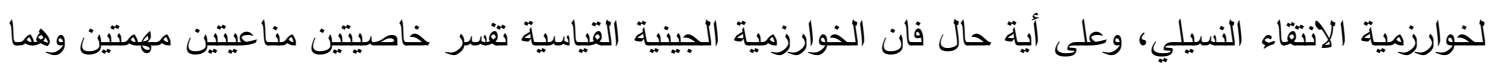
التكاثر النسبي وعملية الطفرة، وربما تتضمن المسألة المراد إيجاد أمثلية لها في بعض الحالية الحات أنمانية أنماطاً ذاتية ومتغيرة مع تغير عامل الزمن في المسألة[10].

خطوات نظرية الانتقاء النسيلي المستخدمة في البحث موضحة كالآتي:

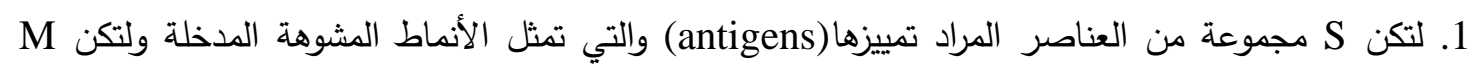
مجموعة من العناصر المراد المقارنة معها (Antibodies) والتي تمثل الأنماط الأصلية للحروف الانكليزية.

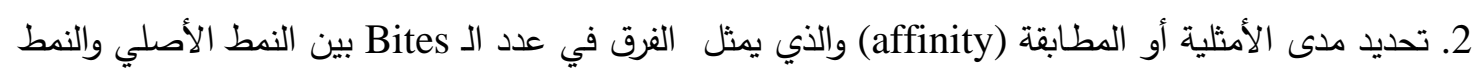

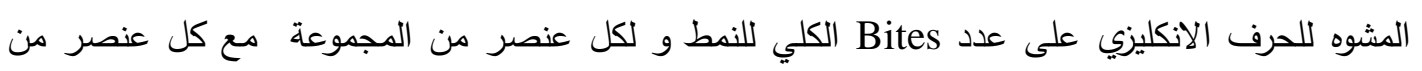
المجموعة M وانتقاء من العناصر من المجموعة S حسب معيار للأمثلية.

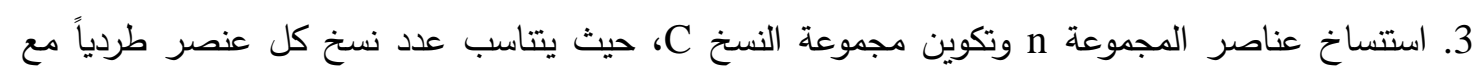
مدى الأمثلية الذي يمثل قيمة معينة خاصة لكل نمط للحرف الانكليزي الأصلي (كلما كانت أمثلية العنصر عالية، يزداد عدد النسخ المكونة منه و العكس صحيح). 4. عمل طفرة (hyper mutation) من نوع (bit inversion) في قيمة كل عنصر من المجموعة C وتكوين

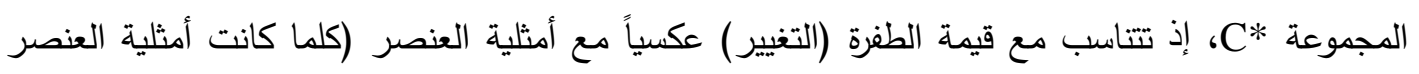
عالية، يقل التغيير الحاصل عليه و العكس صحيح).

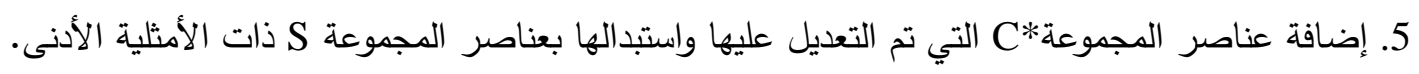
6. تكرار العملية إلى أن يتحقق شرط معين (الانتهاء من تمييز جميع الإدخالات).

9rtificial Immune Network (AIN) الثبكة المناعية الاصطناعية

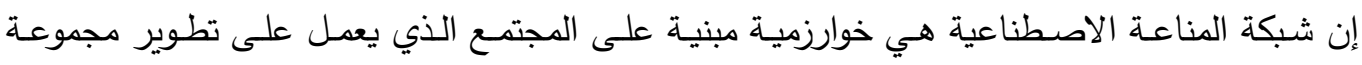

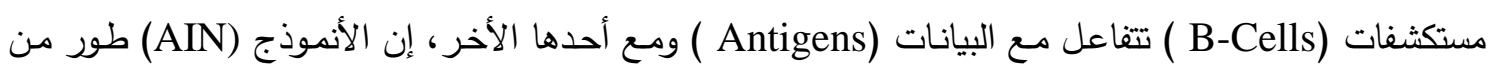

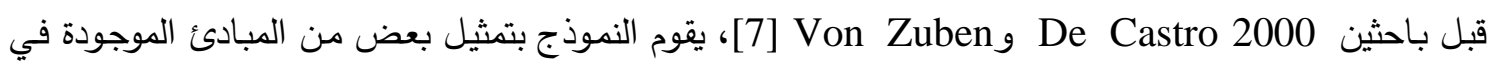

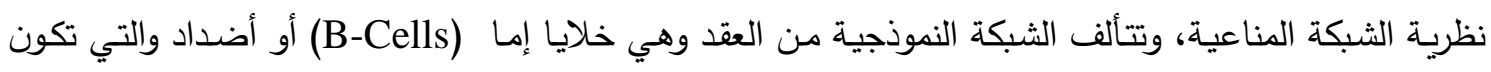
مرتبطة بالحافات لتشكل أزواج العقدة يتم تخصيص قيمة وزن أي (قوة الاتصال) لكل حافة للإشارة إلى التشابه 
بين عقدتين وهكذا فان الثبكة التي تتشكل أثناء التدريب تقدم على هيئة رسم بياني والثكل (1) يوضـح المستقبلات التي تمييز الأنماط [10].

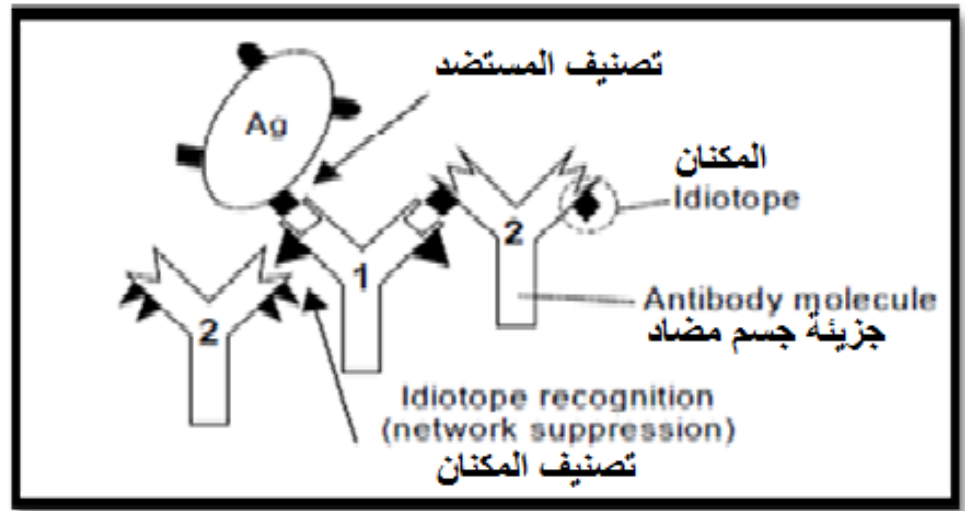

الثكل (1). يوضح المستقبلات التي تمييز المستضدات

10. خطوات نظرية الشبكة المناعية الاصطناعية(AIN)

لتكن (P) مجموعـة مـن الأنمـاط المشوهة للحرف الانكليزي، فإن الخوارزميـة الأساسية لنظريـة الثـبكة المناعية الاصطناعية المستخدمة في البحث تعمل على النحو الآتي:-

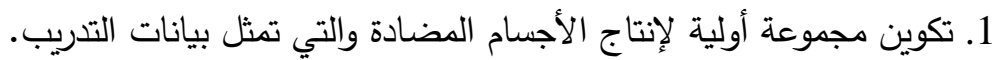

2. لكل نمط مستضد في (P) نطبق نظرية الانتقاء النسيلي clonal selection algorithm المذكورة سابقاً في

الفقرة (8)

3. نكمل خطوات خوارزمية الثبكة المناعية الاصطناعية بحيث لا نحصل على مجموعة من خلايا الذاكرة

$$
\text { التي سوف ترجع مجموعة من خلايا الذاكرة (M*) }
$$

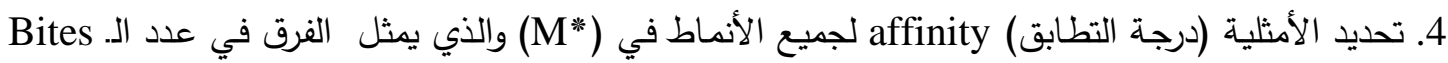
بين النمط الأصلي والنمط المشوه للحرف الانكليزي على عدد Bites الكلي للنمط. 5. حذف كل واحد من الأفراد (الأنماط الناتجة المشوهة) في (M*3) الذي أمثليته تكون اقل من عتبة معينة يتم تحديدها حسب نمط كل حرف انكليزي أصلي، الغرض من هذه العملية هو القضاء على (threshold) التكرار في الثبكة بواسطة كبت عناصر التمييز الذاتي. 6. دمج الأنماط المتبقية من الخطوة السابقة مع الأنماط الباقية التي وجدت لكل نمط مستضد، لوند وهذا سيؤدي إلى

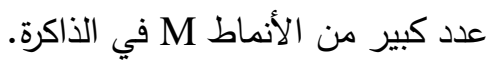

7. تحديد نسب الأمثلية affinity في الجيل M وكبت الجميع ماعدا واحد من العناصر الذاتية التمييز • وهذا

سيؤدي إلى تقليل عدد الجيل النهائي في خلايا الذاكرة التي تتميز وتتبع التوزيع المتميز للمستضدات. 8. نكرر الخطوات من 2 إلى 8 حتى يتحقق شرط التوقف المحدد مسبقا وهو تمييز الأنماط.

$$
\text { 11. الجانب العملي للبحث }
$$

تم في البحث استخدام إحدى التقنيات الذكائية الاصطناعية وهو النظام المناعي الاصطناعي دIS

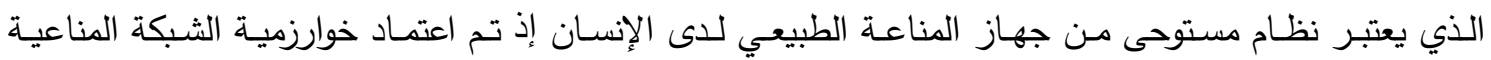

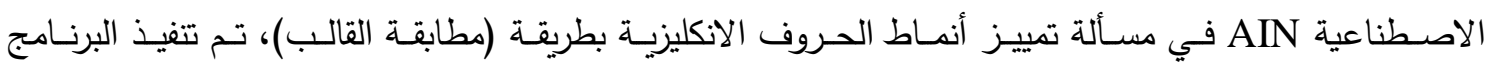


بلغة MATLAB 2008 حيث عند بدأ التنفيذ يتم إدخال الأنماط الأصلية للحروف الانكليزية وخزنها في البرنامج بملف، إن قاعدة البيانات تتضمن تمثيل كل حرف انكليزي على شكل مصفوفة 8 8*8

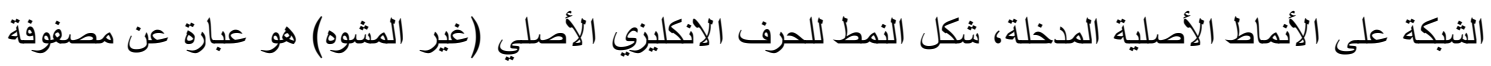
8*8 حيث ذا ذات اللون الأسود تمثل رقم (1) أما bit ذات اللون الأبيض تمثل الرقم (0) ثم يتم تحويل البيانات من النظام الثنائي إلى النظام السـادس عشر ، ثم تتم عملية إدخال الأنماط المشوهة للحروف الانكليزيـة المراد

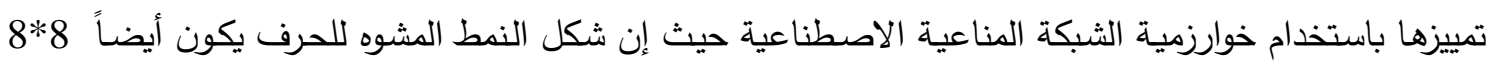
وبالنظام الثنائي ثم تحول إلى النظام السـادس عشر ، نلاحظ الثكل (2) يمثل واجهة إدخـال الأنماط المشوهة

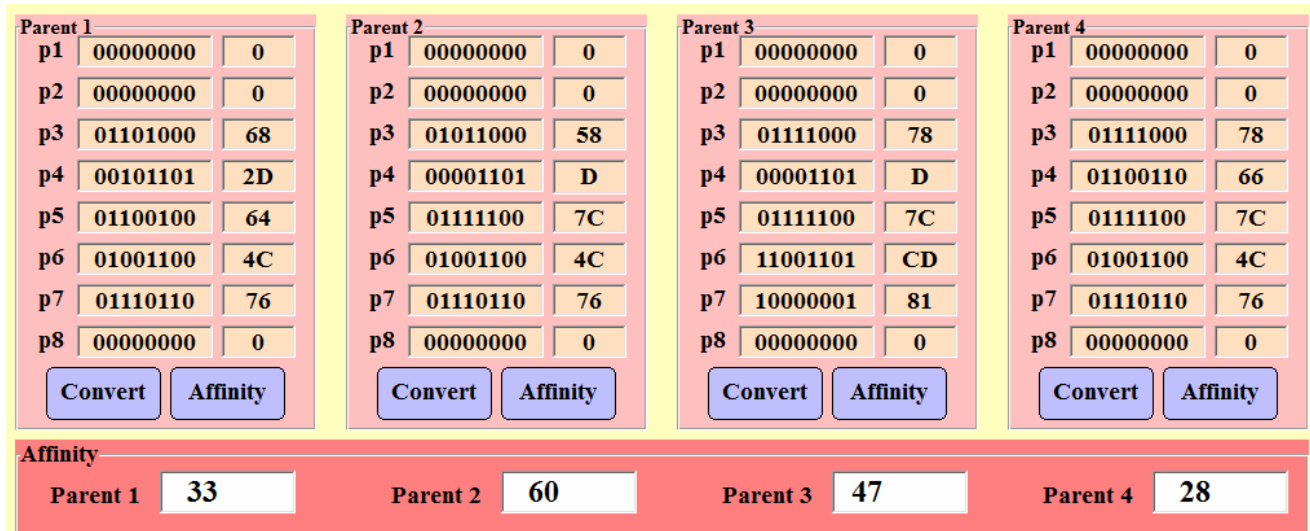

\section{Show Char}

(a) الشكل (2). يمثل واجهة إدخال الأنماط المشوهة للحرف الانكليزي

نلاحظ أن الثكل (3) يمثل واجهة إدخال الأنماط المشوهة للحرف الانكليزي (a) حيث تم تمثلئ تمثيلها بقيم ثنائية (0,1) أما الثكل (4) يمثل واجهة عرض الأنماط المشوهة.

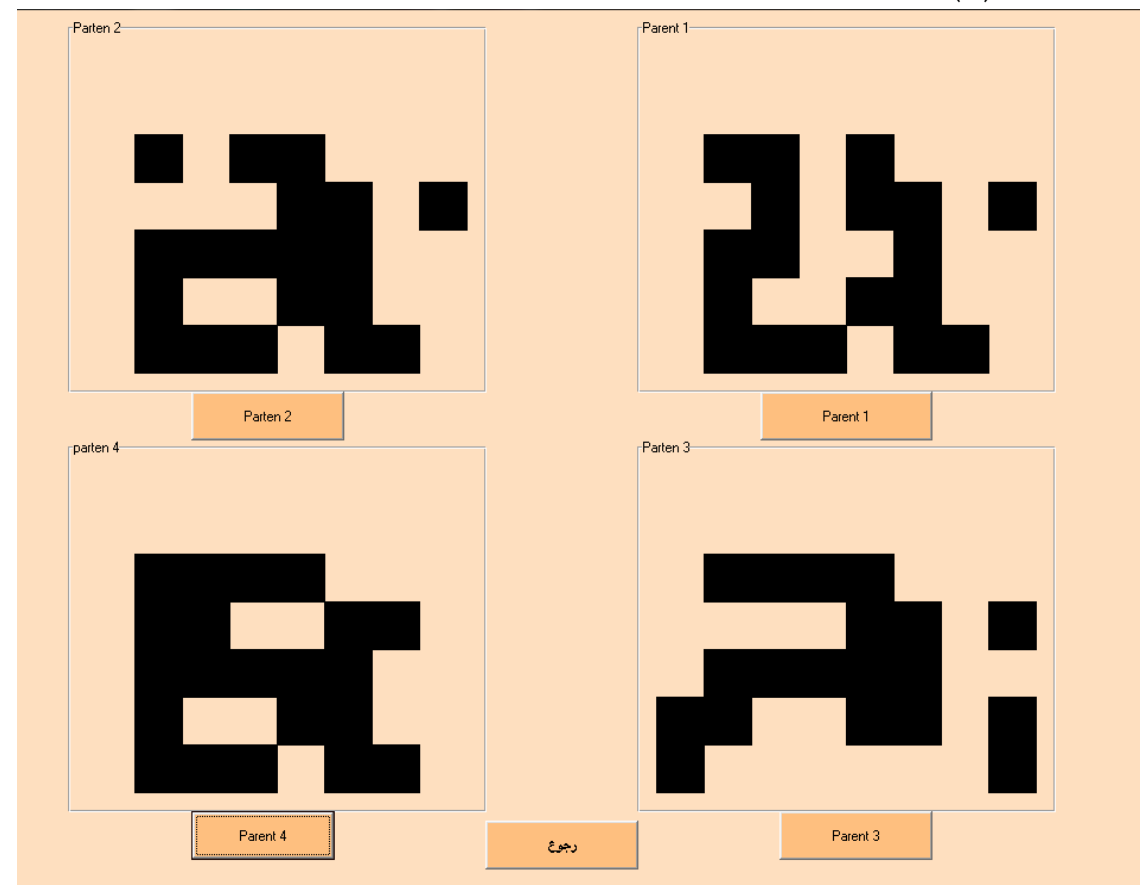




$$
\text { الثكل (4). يمثل واجهة عرض الأنماط المشوهة المدخلة }
$$

ويكون عدد الأنماط المشوهة للحروف الانكليزية المدخلة حسب بيانات الشخص المستخدم للنظام (حسب حاجة .) (userل)

الجدول (1) يمثل عرض معلومـات النمط الذي تم اختياره للحرف الانكليزي المشـوه حيث إن كلمـة

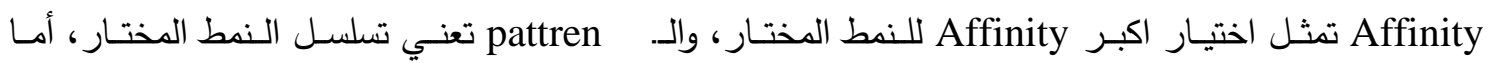
clon_number clon_number= Affinity value $/ 10$

أما mutation_number تعني مقدار الطفرة الناتجة من المعادلة التالية: (1) mutation_number $=$ No. of All Bites - Affinity value

الجدول (1). يمثل معلومات النمط الذي تم اختياره

\begin{tabular}{|c|c|}
\hline Pattern information's & values \\
\hline Affinity & 60 \\
\hline Pattern & 2 \\
\hline Cloun_number & 6 \\
\hline Mutation_number & 4 \\
\hline
\end{tabular}

الثكل (5) يمثل عرض النمط المشوه بعد إجراء عملية معالج له وهي تكرار خطوات الخوارزمية مرة

\section{Parten 2}

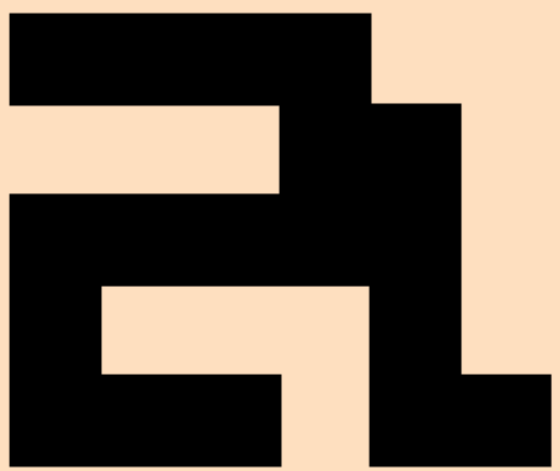

Parten 2

الثكل (5). يمثل عرض النمط الذي تم إجراء عملية معالجة له

الثكل (6) يوضح النمط للحرف الانكليزي بعد الانتهاء من إجراء عمليات المعالج عليه. 


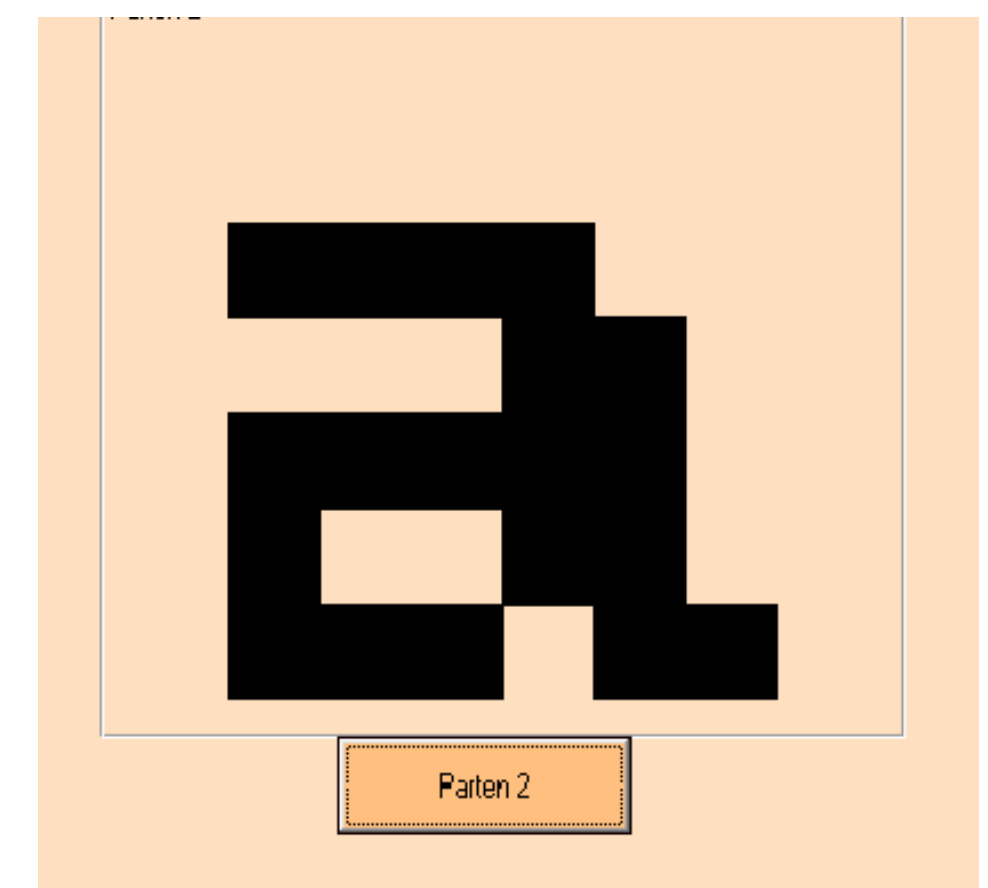

الواجهة (6). تمثل النمط المختار بعد الانتهاء من إجراء عمليات المعالج عليه.

12. الاستنتاجات

من خلال النتائج التي تم التوصل إليها نلاحظ أن الأنماط الناتجة اقرب إلى النمط الأصلي وهي نسبة

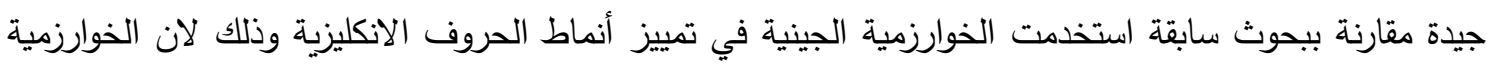

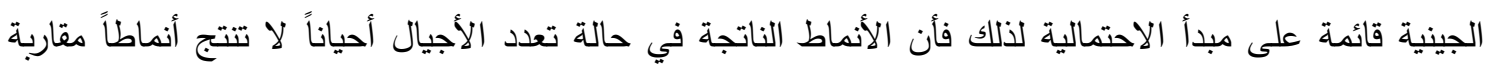
للنمط الأصلي للحرف الانكليزي المدخل أو تتوقف الخوارزمية الجينية لتكرارات محددة دون الوصول التهلية إلى أنماط

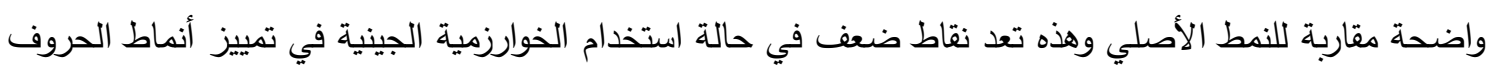
الانكليزية.

أما بالنسبة لخوارزمية الثبكة المناعية الاصطناعية (AIN) فان عملية حذف النمط المشوه الذي يمتلك

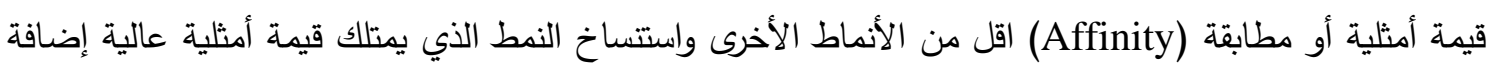

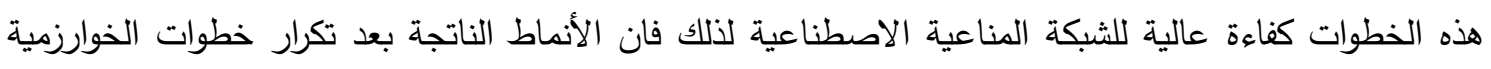
تكون اقرب إلى النمط الأصلي، لو تمت مقارنة نتائج عملية تمييز أنماط الحروف الانكليزية (طريقة مطابقة القالب)

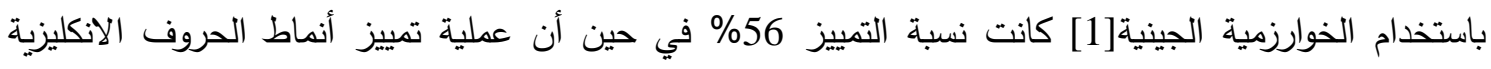

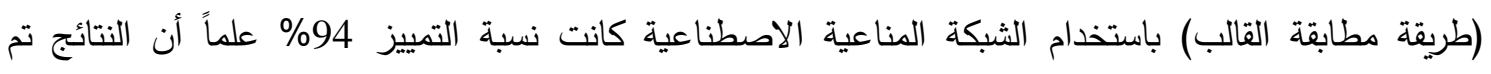

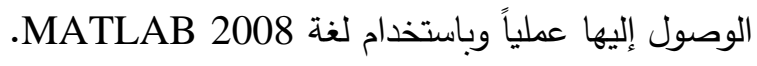


المصادر

البدراني، مها عبد الإله، (2007)، " استخدام الخوارزمية الجينية في تطابق أنماط الحرف الانكليزي"،

مجلة التربية والعلم، جامعة الموصل.

السراج، رشا غانم سعيد، (2012)، " تصنيف وثائق هندسة البرمجيات بالاعتماد على النظام المناعي

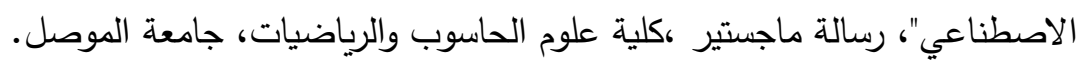

السيلاني إسماعيل علي، (2010)، "تصميم وتتفيذ نظام مناعي اصطناعي لكثف هجوم الفيض"، وسلة رسالة

ماجستير ،كلية علوم الحاسبات والرياضيات، جامعة الموصل.

[4] Ada, G.L. \& Nossal, G.J.V. (1987), "The Clonal Selection Theory”, Scientific American, 257(2), pp. 50-57.

[5] Carter, J. H. (2000), "The Immune System as a Model for Pattern Recognition and Classification", Journal of the American Medical Informatics Association, 7(3), pp. 28-41.

[6] C.-L. LIU. " Normalization-cooperated Gradient Feature Extraction for Handwritten Character Recognition ", IEEE Trans. Pattern Analysis and Machine Intelligence, vol. 29, 2007, p. 1465-1469.

[7] C.-L. LIU, C.Y. SUEN. "A new benchmark on the recognition of handwritten Bangla and Farsi numeral characters in Pattern Recognition", vol. 42, 2009, p. 3287-3295.

[8] C.-L. LIU, H. SAKO. "Class-specific feature polynomial classifier for pattern classification and its application to handwritten numeral recognition in Pattern Recognition", vol. 39, 2006, p. 669-681.

[9] De Castro, L.N., \& Von Zuben, F.J., (2001), "aiNet: An Artificial Immune Network for Data Analysis", In Data Mining: A Heuristic Approach, H. A. Abbass, R. A. Sarker, and C. S. Newton (Eds.), Idea Group Publishing, USA, Chapter XII, pp. 231-259.

[10] De Castro, L.N. \& Von Zuben, F.J. (2000), "The Clonal Selection Algorithm with Engineering Applications”, GECCO'00 - Workshop Proceedings, pp. 3637.

[11] Forrest, S., Javornik, B., Smith, R.E. \& Perelson, A.S. (1993), "Using Genetic Algorithms to Explore Pattern Recognition in the Immune System", Evolutionary Computation, 1(3), pp. 191-211.

[12] Hofmeyr S. A. \& Forrest, S. (2000), "Architecture for an Artificial Immune System", Evolutionary Computation, 7(1), pp. 45-68.

[13] Julie Greensmith, 2003, "New Frontiers For An Artificial Immune System", School of Computing, University of Leeds, Copyright Hewlett-Packard Company.

[14] L. L. MA, C.-L. LIU. On-line handwritten Chinese character recognition based on nested segmentation of radicals, in "Proc. 1st CJKPR", vol. 2, 2009, p. 929933.

[15] L. N. de Castro and J. Timmis, 2002, "Artificial Immune Systems: A Novel Paradigm to Pattern Recognition", Computing Laboratory, University of Kent at Canterbury.

[16] T. H. SU, T. W. ZHANG, D. J. GUAN, H. J. HUANG." Off-line recognition of realistic Chinese handwriting using segmentation-free strategy in Patten Recognition", vol. 42, 2008, p. 167-182. 